\title{
LUXAÇÃO LATERAL E INTRUSIVA ASSOCIADA A FRATURA CORONÁRIA EM DENTES PERMANENTES DE PACIENTE PEDIÁTRICO: RELATO DE CASO CLÍNICO
}

SIDE AND INTRUSIVE LUXATION ASSOCIATED WITH CORONARY FRACTURE IN PERMANENT TEETH OF A PEDIATRIC PATIENT: CLINICAL CASE REPORT

\section{Madelaine Torres da Silva}

Cirurgiã-Dentista em estágio probatório para o Mestrado em Odontologia da Universidade Federal Fluminense

\section{Suelen Cristina Sartoretto}

Professora da disciplina de Cirurgia Bucal da Faculdade de Odontologia da Universidade Iguaçu - UNIG e Professora da Pós-Graduação da Universidade Veiga de Almeida - RJ

\section{José Calasans Maia}

Professor da disciplina de Ortodontia da Universidade Federal Fluminense - UFF

\section{Mônica Diuana Calasans Maia}

Professora titular da disciplina de Cirurgia Oral Menor da Universidade Federal Fluminense - UFF

\section{Vittorio Moraschini Filho}

Professor da Pós-Graduação (Mestrado / Doutorado) da Universidade Veiga de Almeida $-\mathrm{RJ}$

Instituição: Faculdade de Odontologia da Universidade Federal Fluminense

Categoria: Relato de Caso 
COPYRIGHT @ 2020 INTERNATIONAL JOURNAL OF SCIENCE DENTISTRY | AVAILABLE ONLINE http://www.periodicos.uff.br/index

Informações do autor principal: Madelaine Torres da Silva, Rua Lourenço Furtado de Mendonça, no 227, CEP 24421-520, São Gonçalo-RJ. madtorres.mt@gmail.com

\section{RESUMO}

As lesões dentárias traumáticas em pacientes pediátricos são condições relativamente comuns na Odontologia. A avaliação e o diagnóstico corretos são determinantes para o sucesso do tratamento a longo prazo. Este relato descreve o caso de uma paciente pediátrica que sofreu uma queda da própria altura que ocasionou luxação lateral associada a luxação intrusiva, além de fratura da tábua óssea alveolar vestibular e fratura coronária dos elementos envolvidos, exigindo um manejo clínico delicado e efetivo. Em dois anos de acompanhamento a paciente não apresentou nenhuma complicação, exibindo resultados clínicos satisfatórios com a conduta terapêutica aplicada.

Palavras-chave: Traumatismo dentário, emergências, osso, dentes, periodontal. 


\section{ABSTRACT}

Traumatic dental injuries in pediatric patients are relatively common conditions in dentistry. Correct medical evaluation and diagnosis are critical to long-term treatment success. This report describes the case of a pediatric patient who suffered a fall from his own height that caused lateral dislocation associated with intrusive dislocation, in addition to fracture of the vestibular alveolar bone plate and coronary fracture of the involved elements requiring a delicate and effective clinical management.

In two years of follow-up, the patient did not present any complications, showing satisfactory clinical results with the applied therapeutic approach.

Key words: Dental trauma, emergencies, bone, teeth, periodontal. 


\section{INTRODUÇÃO}

Os traumatismos dentários são considerados frequentes tanto na dentição decídua quanto na dentição mista. Os traumas dentários podem ser classificados como lesões ao tecido dental e à polpa, que pode ser envolvendo apenas esmalte, esmalte e dentina com ou sem exposição pulpar, podendo ainda envolver o cemento e/ou a raiz; lesões ao tecido periodontal, classificadas como concussão, subluxação, luxação intrusiva, luxação extrusiva, luxação lateral, avulsão e fratura radicular retida no alvéolo. Além disso, as lesões podem acometer o tecido ósseo, e são classificadas como cominução do osso alveolar, fratura da parede alveolar, fratura do processo alveolar $\mathrm{e}$ fraturas de maxila e mandíbula. (FONSECA RJ, WALKER RV et al, 2013)

A avaliação e o diagnóstico correto do caso são um verdadeiro desafio ao profissional, pois o tratamento inicial de emergência de lesões dentárias traumáticas e a conduta terapêutica adotada, são determinantes para o sucesso do tratamento a longo prazo. (MYERS, 2019). A maioria dos traumas ocorre de forma corriqueira, como quedas e colisões. (ANDREASEN JO, ANDREASEN FM,1991) Entretanto, a maior participação das crianças em atividades esportivas, o aumento da violência e os acidentes de trânsito também contribuem para o aumento dos casos. (TRAEBERT J, CLAUDINO D, 2012)

Os casos de luxação lateral são clinicamente caracterizados por um posicionamento da coroa dentária no sentido palatino e da raiz no sentido vestibular. Existe, portanto, zonas de intensa compressão do ligamento periodontal, polpa dentária e osso adjacente. (ANDREASEN FM,1985). Os elementos dentários que sofreram luxação lateral podem evoluir com anquilose, e, ao teste de percussão, produzem um som alto e metálico. Os testes de sensibilidade, com frequência, são negativos. $O$ tratamento consiste em realizar o reposicionamento suave do dente para sua posição original e estabilização durante quatro semanas. O tratamento endodôntico é indicado para evitar reabsorção radicular. (FLORES MT, ANDERSSON L et al, 2007).

A luxação intrusiva é caracterizada pelo deslocamento forçado do dente para o interior do alvéolo, provocando cominução ou fratura da tábua óssea alveolar. (ANDREASEN JO,1985). Normalmente os dentes que sofreram luxação intrusiva não 
apresentam mobilidade e o teste de percussão emite, também, um som alto e metálico. Os testes de sensibilidade são, em geral, negativos, e, em dentes em desenvolvimento, com ápices abertos, há possibilidade de revascularização. O tratamento varia de acordo com o estágio de formação das raízes, sendo indicado desde o reposicionamento ortodôntico ou cirúrgico no caso de dentes completamente desenvolvidos, até o acompanhamento por aproximadamente três semanas para aguardar o reposicionamento espontâneo, em caso de dentes com as raízes em formação. (FLORES MT, ANDERSSON L et al, 2007).

Baseado nessas informações, este trabalho tem por objetivo relatar um caso clínico de luxação lateral e intrusiva, associada a fratura da tábua óssea vestibular alveolar e fratura coronária em uma paciente pediátrica sob acompanhamento de dois anos.

\section{RELATO DO CASO}

L.M, sexo feminino, 8 anos de idade foi encaminhada para a Faculdade de Odontologia da Universidade Federal Fluminense após sofrer uma queda da própria altura. Ao exame extraoral, foi observado corte na região mentoniana que foi tratado através de sutura epidérmica. No exame intraoral, foram observadas lacerações, edema e sangramento no tecido gengival. Os elementos 12, 11 e 21 encontravam-se intruídos. Após consentimento dos pais, a criança recebeu atendimento emergencial de assepsia e debridamento das feridas. No exame tomográfico foram observadas fraturas complicadas da porção coronária, envolvendo esmalte e dentina nos elementos $11 \mathrm{e}$ 21 , luxação lateral nos mesmos elementos e também no elemento 22 , além de fratura da tábua óssea vestibular na região do 21.

A conduta terapêutica adotada consistiu em realizar o delicado reposicionamento dos elementos dentários com auxílio de fórceps e redução da tábua óssea vestibular, seguida de sutura das papilas interdentais. O aparelho ortodôntico foi instalado e mantido durante 28 dias para manutenção do correto posicionamento dentário. Os responsáveis pela paciente foram orientados quanto aos cuidados pós-operatórios e dieta líquida e pastosa durante 28 dias. Após uma semana do trauma, a paciente foi submetida ao tratamento endodôntico dos dentes 12,11,21 e 22 com trocas semanais 
de hidróxido de cálcio durante quatro semanas. Por fim foi realizado o tratamento restaurador com resina composta.

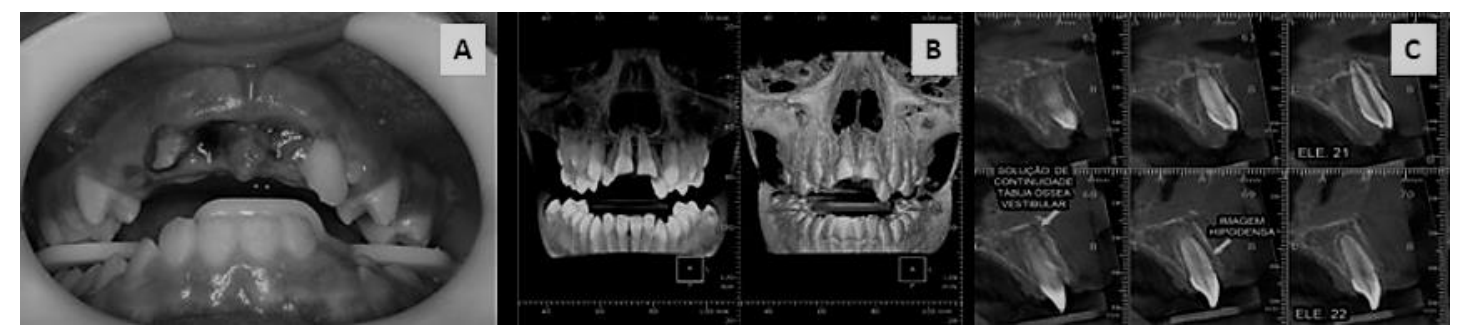

Figura 1. (A) Foto intraoral da condição inicial, observe a intrusão dos elementos 11, 12 e 21; (B) aspecto tomográfico inicial, observe a luxação lateral dos elementos 11 e 21; (C) corte parassagital na região do 21 , observe a fratura da tábua óssea vestibular apontada com a seta.

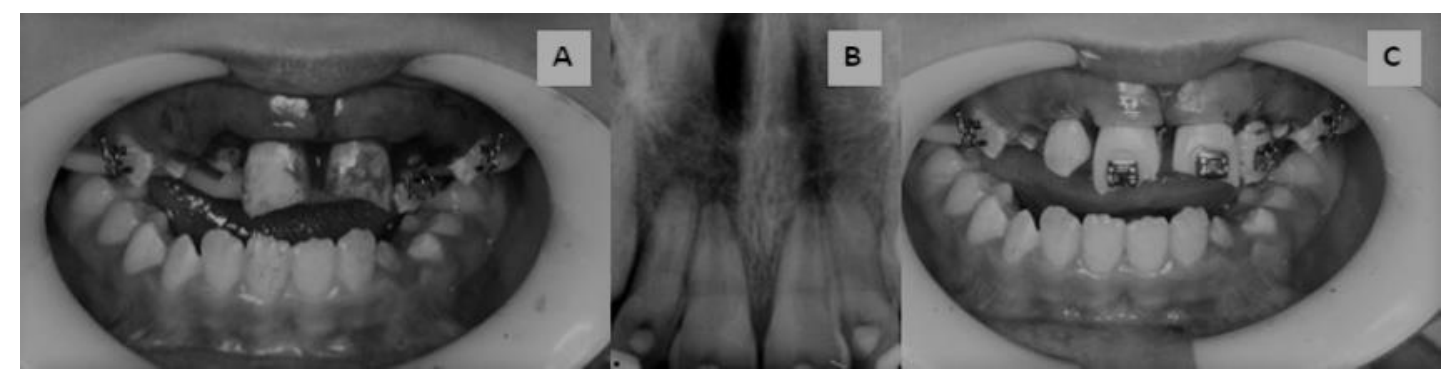

Figura 2. (A) Vista intraoral do reposicionamento dentário pós-operatório; (B) imagem radiográfica após o reposicionamento; (C) instalação dos dispositivos ortodônticos e sutura das papilas interdentais.

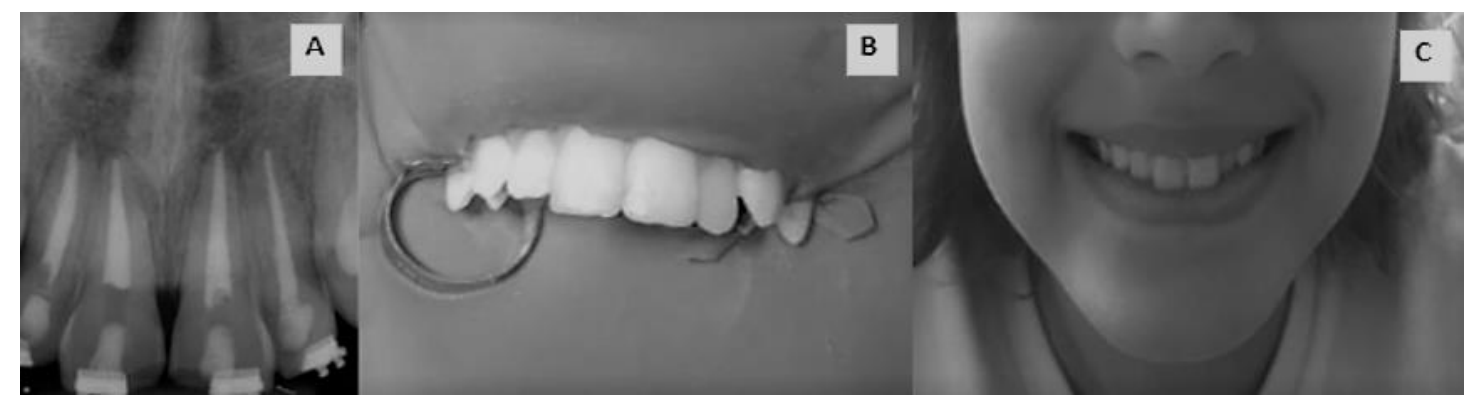

Figura 3. (A) Aspecto radiográfico após tratamento endodôntico nos elementos 11, 12, 21 e 22; (B) aspecto clínico após tratamento restaurador; (C) acompanhamento clínico após dois anos. 


\section{DISCUSSÃO}

O trauma dentário é uma condição relativamente comum, sendo a avaliação e o diagnóstico precisos os passos fundamentais para o correto tratamento.

É importante obter o máximo de informações acerca da história da lesão, como por exemplo, onde, quando e como ocorreu, pois essas informações ajudarão a entender a intensidade e a mecânica do trauma. O exame clínico deve incluir a avaliação, não só das estruturas dentárias, mas também da face e da cabeça, priorizando assim as lesões mais graves. Além disso, a documentação do caso através de registros fotográficos iniciais durante $o$ tratamento e durante a fase de acompanhamento é de extrema importância. (MYERS, 2019). Exames de imagem como radiografias e tomografias são ferramentas imprescindíveis para a realização de um diagnóstico completo, elaboração do plano de tratamento e tomada de decisão.

As abordagens de tratamento para as luxações laterais e intrusivas são variáveis, e devem analisar, por exemplo, se há fratura da tábua óssea alveolar e os resultados de testes de sensibilidade pulpar. Embora os testes iniciais de sensibilidade pulpar sejam importantes, eles podem ser inconclusivos por até três meses após o trauma. (BARKIN PR, 1973). Feiglin (FEIGLIN B, 1996) relatou em seus estudos que os dentes traumatizados devem estar sob observação por até cinco anos após o trauma, sendo o tratamento endodôntico iniciado somente após indícios clínicos e radiográficos de patologia apical.

Podemos entender que a viabilidade da polpa dentária após as lesões traumáticas varia de acordo com a classificação do trauma, sendo a resposta do teste inicial muitas vezes inconclusiva. Os testes de sensibilidade comumente utilizados avaliam apenas a resposta neural ao estímulo, entretanto a vascularização pode ser o fator mais importante associado a capacidade de viabilidade pulpar. Métodos de testes alternativos como oximetria de pulso (Caldeira CL, Barletta, FB, et al, 2016) e fluxometria doppler ultrassônica ou a laser (OLGART L, GAZELIUS B, LINDH-STROMBERG,U 1988) já foram relatados e mais estudos devem ser realizados para otimizar a avaliação pulpar. 
As fraturas ósseas decorrentes de traumas dentários normalmente acometem o osso alveolar podendo se estender para o osso adjacente, apresentando clinicamente mobilidade e deslocamento do seguimento fraturado, além de alterações oclusais. $O$ tratamento consiste na redução correta das estruturas ósseas e estabilização dentária durante quatro semanas. (FLORES MT, ANDERSSON L et al, 2007). Dependendo da gravidade do caso, a fixação interna rígida para estabilização dos segmentos ósseos do rebordo alveolar deslocados pode ser necessária (FONSECA RJ, WALKER RV et al, 2013).

Existem diversos tipos de técnicas de imobilização dentária descritas na literatura. Atualmente entende-se que o material utilizado deve ser passivo e flexível, permanecendo o mínimo de tempo possível em boca e permitindo a mobilidade fisiológica dos dentes, alimentação e higiene do paciente. (FILIPPI A, VON ARX T, LUSSI A, 2002). No presente caso, devido ao trauma severo que ocasionou intrusão dos elementos dentários 11,12 e 21, associada a luxação lateral desses elementos e do elemento 22 , além de fratura da parede vestibular do alvéolo na região do dente 21 , optou-se pelo reposicionamento dos dentes seguido de imobilização com fio de aço durante 28 dias. Optou-se pela realização do tratamento endodôntico dos dentes traumatizados devido a extensão do trauma e por fim o tratamento restaurador das fraturas coronárias.

É importante que o dentista clínico tenha conhecimento dos tipos de lesões traumáticas, suas associações e suas abordagens, desde o contato inicial com o paciente traumatizado - que exige um manejo diferenciado, pois o paciente normalmente está acompanhado de familiares que estão sob uma situação de estresse, até os tratamentos que podem ser realizados de imediato, tendo em vista que são situações de emergência.

\section{CONCLUSÃO}

Dois anos após o tratamento a paciente não apresentou nenhuma complicação, indicando resultados clínicos satisfatórios através da conduta terapêutica adotada. A mesma permanece em acompanhamento clínico. 


\section{REFERÊNCIAS}

1. Fonseca RJ et al, Oral and Maxillofacial Trauma, 4르. Ed. St. Louis, Missouri, Elsevier 2013.

2. Garry L. Myers. Evaluation and diagnosis of the traumatized dentition. Dental Traumatology. 2019;35:302-308.

3. Andreasen JO. Challenges in clinical dental traumatology.Endodont Dent Traumatol. 1985;1:45-55.

4. Trabert J, Claudino, D. Epidemiologia do Traumatismo Dentário em Crianças: A Produção Científica Brasileira. Pesq Bras Odontoped Clin Integr, 2012; 12(2):263-72, abr./jun., 2012.

5. Andreasen, JO, Andreasen FM. Traumatismo dentário: soluções clínicas, São Paulo: Panamericana, 1991.

6. Flores MT, Andersson L, et al. Guidelines for the management of traumatic dental injuries. I. Fractures and luxations of permanent teeth. Dental Traumatology. 2007; 23: 66-71.

7. Barkin PR. Time as a factor in predicting the vitality of traumatized teeth. J Dent Child. 1973;40:188-92.

8. Feiglin B. Dental pulp response to traumatic injuries: a retrospective analysis with case reports. Endod Dent Traumatol. 1996;12:1-8.

9. Caldeira CL, Barletta FB, et al. Pulse oximetry: a useful test for evaluating pulp vitality in traumatized teeth. Dent Traumatol. 2016;32:385-9.

10. Olgart L, Gazelius B, Lindh-Stromberg U. Laser doppler flowmetry in assessing vitality in luxated permanent teeth. Int Endod J. 1988;21:300-6.

11. Filippi A, von Arx T, Lussi A. Comfort and discomfort of dental trauma splints - a comparison of a new device (TTS) with three commonly used splinting techniques. Dent Traumatol 2002;18:275-80. 
